\title{
Huevo, larva en primer estadio y aparato genital femenino de la mariposa Chabuata castanea (Lepidoptera: Noctuidae)
}

\author{
Marcela A. Rodríguez ${ }^{1}$ \& A.O. Angulo ${ }^{1,2}$ \\ 1 Universidad de Concepción. Facultad de Ciencias Naturales y Oceanográficas. Departamento de Zoología, Casilla \\ 160-C, Concepción, Chile; marcrodr@udec.cl \\ 2 aangulo@udec.cl
}

Recibido 11-V-2001. Corregido 19-V-2006. Aceptado 12-I-2007.

\begin{abstract}
Egg, first larval stage and female genitalia of the moth Chabuata castanea (Lepidoptera: Noctuidae). Egg, first larval stage, and female genitalia of the widely distributed moth Chabuata castanea are described, based on material from Talcahuano, VIII region, Chile. Egg microestructures are illustrated with scannig electron microscope images which show that egg morphology allows identification to species level. Rev. Biol. Trop. 55 (2): 659-664. Epub 2007 June, 29.
\end{abstract}

Key words: Chabuata castanea, Hadeninae, inmature stage, Talcahuano, Chile.

Lasionycta castanea fue descrita por Koehler (1961), además coloca a la especie carneago en el mismo género, ya que comparten caracteres. Según Poole (1989) las especies son colocadas en distintos géneros, carneago en Chabuata y castanea colocada en "Lasionycta de autores". Finalmente León et al. (2005) ubica a la especie castanea en el género Chabuata Walker, 1858.

Chabuata castanea (Köhler, 1961) es un lepidóptero nóctuido perteneciente a la subfamilia Hadeninae, considerado en Chile, plaga de importancia agrícola (Parra et al. 1986). Existe información acerca del imago entregada (Parra et al. 1986) donde se encuentra una diagnosis del macho y hembra de esta especie además de las estructuras genitales del macho. Este trabajo incluye claves, donde esta especie se identifica tanto por caracteres externos del adulto, como caracteres de los genitales del macho.

La distribución conocida de la especie es en Chile a partir de Santiago (Región Metropolitana) hasta Los Angeles (VIII región) y en Argentina en Paso Flores, Neuquén.
Las larvas conocidas de las especies del género Chabuata, se alimentan de lechuga y alfalfa según Pastrana (2004) por lo cual, el conocimiento de los estados preimaginales de éstos, son muy importantes. El objetivo de este trabajo es completar el conocimiento de esta especie mediante la descripción del huevo y larva de primer estadio, del mismo modo es entregada la descripción de la estructura genital de la hembra, esto último, para complementar la diagnosis de la especie en cuestión para, en un futuro, incluirla en una clave para los nóctuidos chilenos.

\section{MATERIALES Y MÉTODOS}

El material utilizado para realizar las descripciones se obtuvo a partir de la ovipostura de un ejemplar adulto, rerecolectado en abril de 1999, en la Ciudad de Talcahuano, VIII región, Chile. El ejemplar se mantuvo en laboratorio, en un frasco plástico con tiras de papel secante, roseado de humedad, obteniéndose los huevos y las larvas de primer estadio. 
Tanto huevo como la larva fueron analizados bajo lupa estereoscópica Carl Zeiss y fotografiados en Microscópio electrónico de Barrido, Autoscanning ETEC de la Universidad de Concepción. Mediante el método de Angulo y Weigert (1977), se obtuvo la preparación del aparato genital de la hembra. La simbología utilizada en la figura 11 (mapa setal), sigue la nomenclatura de Stehr (1987).

\section{RESULTADOS}

Aparato genital de la hembra: apófisis anteriores subiguales en largo a las apófisis posteriores; ductus bursae esclerosado y liso en su porción basal, la porción apical membranosa; bursa copulatrix bisacular; corpus bursae subcónico, membranoso; signa ausente; cervix bursae sacular, digitiforme; lóbulos del ovipositor, fuertes, esclerosados y provistos de fuertes espinas dispersas por toda la estructura (Figs. 1, 2).

Huevo: de $0.05 \mathrm{~mm}$ de alto y $0.52 \mathrm{~mm}$ de diámetro, blanquecinos cuando recién ovipuestos, luego toma una coloración castaño claro a oscuro, producto del desarrollo embrional subsecuente. En vista lateral subesféricos, con marcadas ornamentaciones que se pierden hacia la base que es plana y lisa. En vista polar (Fig. 3) se observan 21 costas longitudinales, las que

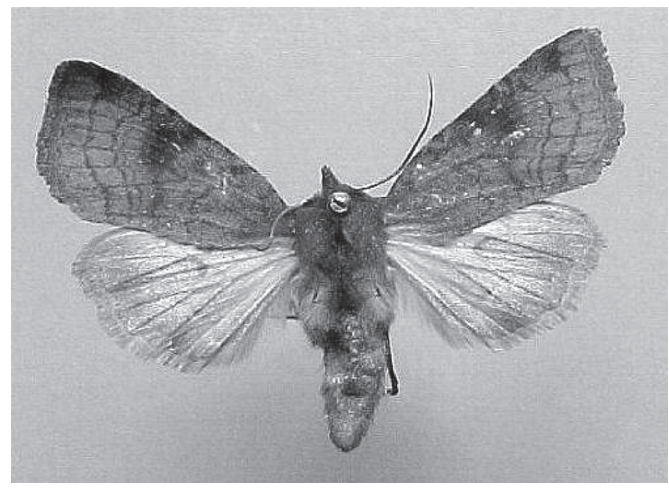

Fig. 1. Vista dorsal adulto hembra de Chabuata castanea.

Fig. 1. Dorsal view, adult female Chabuata castanea.

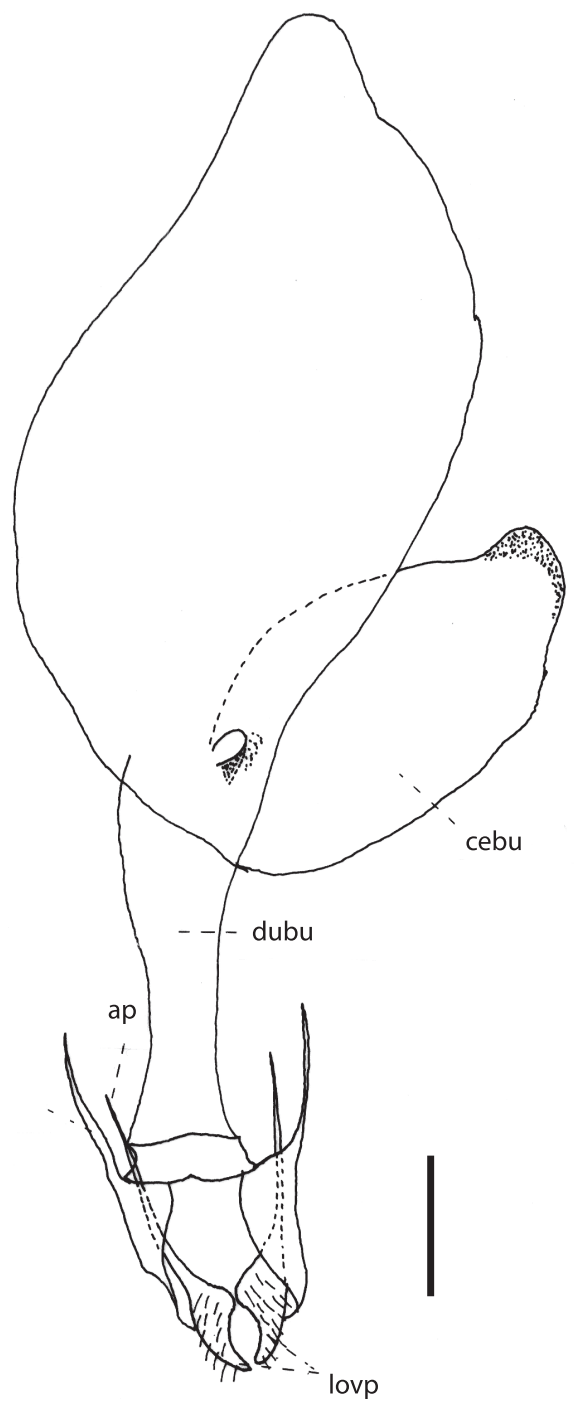

Fig. 2. Aparato genital de la hembra (aa: apófisis anteriores; ap: apófisis posteriores; cebu: cervix bursae; cobu: corpus bursae; dubu: ductus bursae; lov: lóbulos del ovipositor).

Fig. 2. Female genitalia (aa: anterior apophysis; ap: posterior apophysis; cebu: cervix bursae; cobu: corpus bursae; dubu: ductus bursae; lov: ovipositor lobules).

son intersectadas por las costas transversales igualmente notorias aunque menos acentuadas, de las 21 costas longitudinales sólo diez llegan hasta la roseta micropilar. Presencia de aeropilas restringidas a las costas longitudinales (Fig. 4), éstas se distribuyen en forma regular 
y desde la mitad del huevo hacia el área polar de éste. El área micropilar (Fig. 3) ubicada en la región polar, presenta la roseta primaria (Fig. 5) constituida por doce celdas, las que están delimitadas por un grueso reborde, que es plano y hundido en relación con la roseta. Roseta secundaria (Fig. 6) constituida por 13 celdas, en las cuales su borde externo es muy poco marcado en alguno de los casos. Fórmula micropilar 12:13.
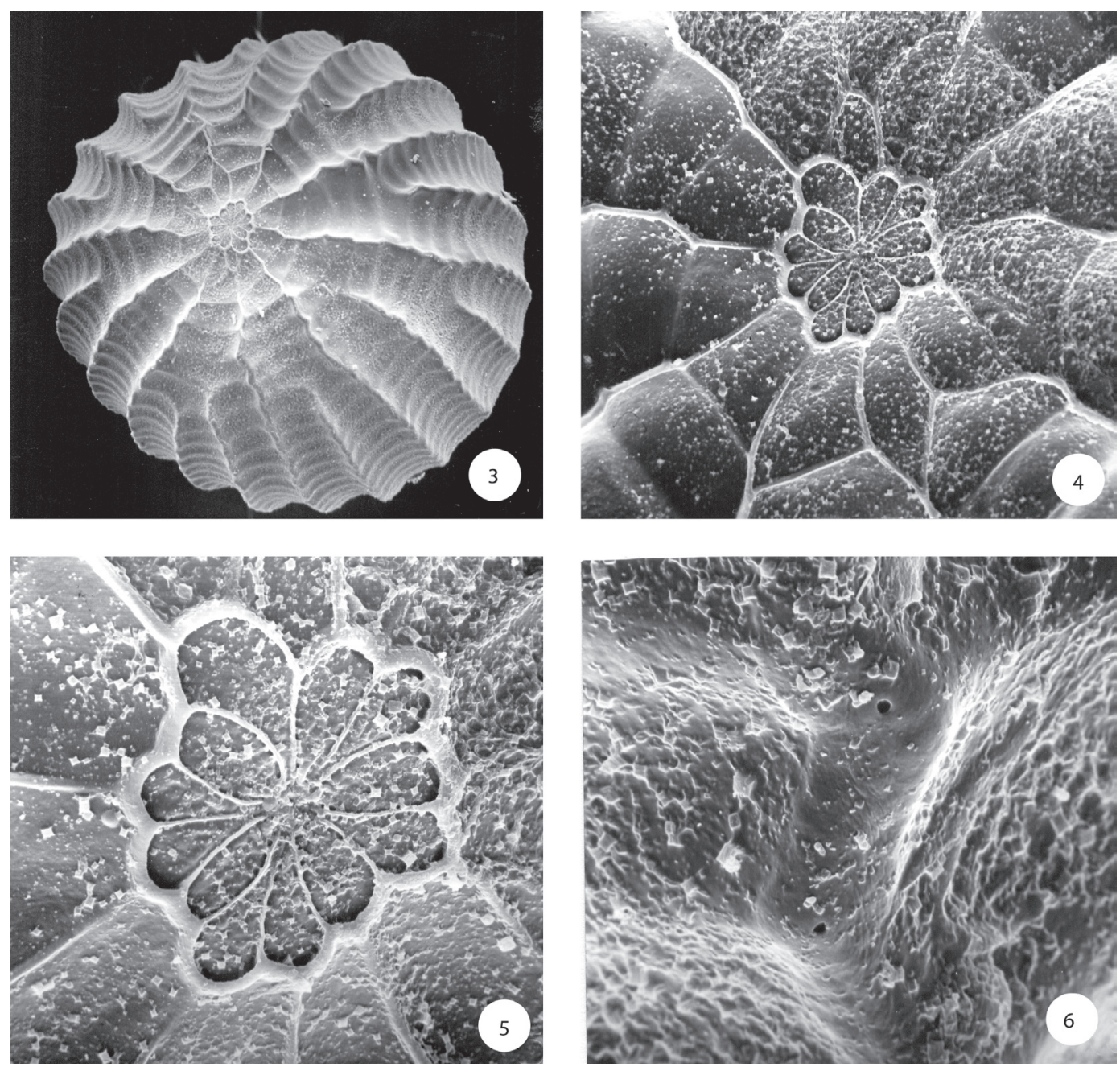

Figs 3-6. Huevo de C. castanea: Fig. 3. Vista polar (80 X); Fig. 4. Aeropilas (1200 X); Fig. 5. micropila, roseta primaria (rp) (600 X); Fig. 6. Roseta primaria (rp) y secundaria (rs) (300 X).

Figs 3-6. Egg of C. castanea: Fig. 3. Polar view (80 X); Fig. 4. Aeropilae (1200 X); Fig. 5. micropila, primary rosette (rp) $(600 \mathrm{X})$; Fig. 6. Primary (rp) and secondary rosettes (rs) (300 X). 
$0.5 \mathrm{~mm}$; cabeza hipognata (Fig. 8), separación de setas frontales $0.03 \mathrm{~mm}$; ocelos (Fig. 8) dispuestos en forma de un dos invertido, la línea que pasa entre los ocelos dos y cinco pasa sobre la base de $\mathrm{S}_{1}$; la base de $\mathrm{SS}_{1}$ intersecta la línea que pasa entre los ocelos cinco y seis; $\mathrm{S}_{2}$ y $\mathrm{S}_{3}$ se encuentran en línea paralela vertical cerca de los ocelos uno y seis respectivamente; $\mathrm{SS}_{2}$ inmediatamente bajo el ocelo 5; $\mathrm{A}_{1}$ y $\mathrm{A}_{2}$ en línea horizontal frente al ocelo dos. Mandíbula (Fig. 9) subcuadrangular, provista de cinco fuertes dientes que presentan crenulado sus bordes internos.

Quetotaxia corporal: (Fig. 11) primer segmento torácico con setas $\mathrm{XD}_{1}, \mathrm{XD}_{2}, \mathrm{D}_{1}$, $\mathrm{D}_{2}, \mathrm{SD}_{1}$ y $\mathrm{SD}_{2}$, sobre el escudo cervical; $\mathrm{L}_{3}$ bajo el primer espiráculo que está en posición oblicua respecto del segmento; $\mathrm{SV}_{1}$ y $\mathrm{SV}_{2}$ sobre la coxa y sus bases en línea partiendo de un mismo tuberculo setígero. Segundo y tercer segmentos torácicos, con bases de $\mathrm{D}_{1}$ y $\mathrm{D}_{2}$ en
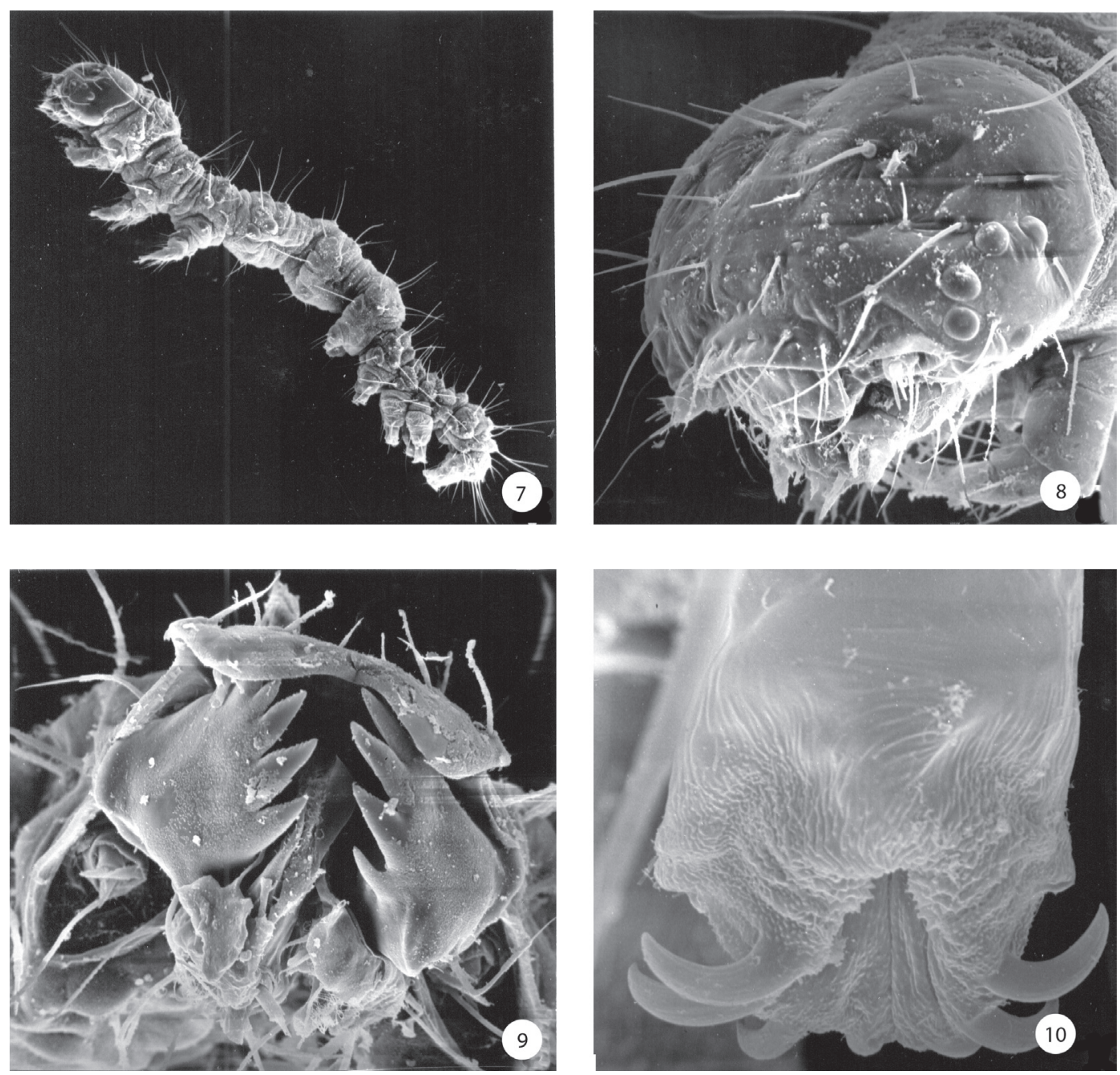

Figs 7-10. Larva de primer estadío de C. castanea: Fig. 7. Vista lateral (40 X); Fig. 8. Cabeza (200 X); Fig. 9. Mandíbula (400 X); Fig. 10. Crochets (2000 X).

Figs 7-10. First stage larva of C. castanea: Fig. 7. Side view (40 X); Fig. 8. Head (200 X); Fig. 9. Mandible (400 X); Fig. 10. Crochets $(2000 \mathrm{X})$. 


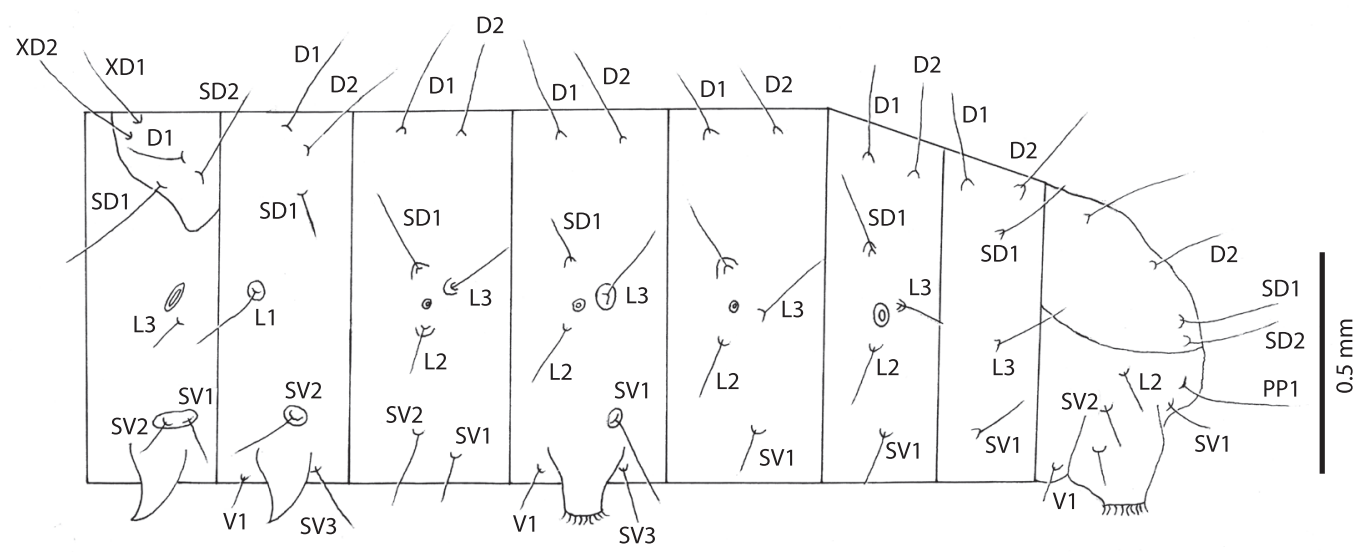

Fig.11. Quetotaxia corporal (Cabeza, área ocular: ant: antena; $\mathrm{A}_{1,2}:$ setas anteriores; $\mathrm{S}_{1,2,3}$ : Setas estematales; $\mathrm{SS}_{1,2}$ : Setas subestematales. Larva: $\mathrm{A}_{1 \mathrm{a} 10}$ : segmentos abdominales 1 al $10 ; \mathrm{XD}_{1,2}:$ setas táctiles del margen anterior de $\mathrm{T} 1 ; \mathrm{D}_{1,2}:$ setas táctiles dorsales; $\mathrm{L}_{1,2,3}$ : setas táctiles laterales; $\mathrm{PP}_{1}$ : seta paraproctal; $\mathrm{SD}_{1,2}$ : setas táctiles subdorsales; $\mathrm{SV}_{1,2,3}:$ setas táctiles supraventrales; $\mathrm{V}_{1}$ : seta ventral; $\mathrm{T}_{1 \mathrm{a} 3}$ : segmentos torácicos 1 al 3).

Fig.11. Body chaetotaxy (head, eye area, ant: antenna; $\mathrm{A}_{1,2}$ : anterior chaetae; $\mathrm{S}_{1,2,3}$ : estematal chaetae; $\mathrm{SS}_{1,2}:$ subestematal chaetae. Larva: $\mathrm{A}_{1 \mathrm{a} 10}$ : abdominal segments 1 to $10 ; \mathrm{XD}_{1,2}$ : tactile chaetae of anterior margin of $\mathrm{T} 1 ; \mathrm{D}_{1,2}:$ dorsal tactile chaetae; $\mathrm{L}_{1,2,3}$ : lateral tactile chaetae; $\mathrm{PP}_{1}$ : paraproctal chaeta; $\mathrm{SD}_{1,2}:$ subdorsal tactile chaetae; $\mathrm{SV}_{1,2,3}:$ supraventral tactile chaetae; $\mathrm{V}_{1}$ : ventral tactile chaeta; $\mathrm{T}_{1 \mathrm{a} 3}$ : thorax segments 1 to 3 ).

línea vertical; $\mathrm{SD}_{1}$ más abajo, está en línea con $\mathrm{D}_{1} ; \mathrm{L}_{1}$ con gran tubérculo setígero; $\mathrm{SV}_{2}$ sobre la coxa, $\mathrm{SV}_{3}$ sobre ella y $\mathrm{V}_{1}$ paralela a $\mathrm{SV}_{3}$. El primer segmento abdominal, con $\mathrm{D}_{1} \mathrm{y}$ $\mathrm{D}_{2}$ con sus bases en línea horizontal; $\mathrm{SD}_{1}, \mathrm{~L}_{3}$, y $\mathrm{L}_{2}$, con notorio tubérculo setígero sobre el espiráculo; $\mathrm{L}_{3}$ sobre y detrás de él y $\mathrm{L}_{2}$ bajo el espiráculo; $\mathrm{SV}_{2}$ y $\mathrm{L}_{2}$ con sus bases alineadas y con $\mathrm{SV}_{1}$ más abajo y al lado de $\mathrm{SV}_{2}$. Segmento abdominal tres a seis con espuripedios desarrollados provistos de ocho crochets en serie uniordinal con penellipse lateral (Fig.10). Las setas $\mathrm{D}_{1}$ y $\mathrm{D}_{2}$ en alineadas, $\mathrm{SD}_{1}$ sobre y delante el espuripedio; $\mathrm{D}_{1}, \mathrm{~L}_{3}$ y $\mathrm{L}_{2}$ con base en línea oblicuamente; $\mathrm{SV}_{1}$ bajo éstas. Segmento abdominal ocho, con $\mathrm{D}_{2}$ y $\mathrm{D}_{1}$ de base paralela, $\mathrm{SD}_{1}$ con base sobre el espiráculo y bajo $\mathrm{D}_{1}$; $\mathrm{L}_{3}$ atrás y al lado del espiráculo, que es dos a tres veces mayor que en los otros segmentos, $\mathrm{L}_{2}$ bajo él; $\mathrm{SV}_{1}$ en línea vertical con el espiráculo. El noveno segmento abdominal con $\mathrm{D}_{1}, \mathrm{D}_{2}$ y $\mathrm{SD}_{1}$ formando un triángulo, $\mathrm{L}_{3}$ de base paralela y vertical a $\mathrm{SD}_{1} ; \mathrm{SV}_{1}$ de base oblicua e inferiormente a $\mathrm{L}_{3}$. El segmento abdominal diez con escudo anal amplio, provisto de las setas $\mathrm{D}_{1}$, $\mathrm{D}_{2}, \mathrm{SD}_{1}$ y $\mathrm{SD}_{2}$ dispuestas siguiendo la línea curva del escudo anal; $\mathrm{SV}_{1}$ débil; $\mathrm{L}_{2}$ sobre la base de la coxa; $\mathrm{SV}_{2}$ y $\mathrm{SV}_{3}$ con sus bases verticalmente ubicadas sobre la coxa; $\mathrm{V}_{1}$ presente, por delante del espuripedio anal.

Material Examinado: siete larvas obtenidas en laboratorio, Mayo 1999.

\section{AGRADECIMIENTOS}

Al personal del Laboratorio de Microscopía Electrónica de la Universidad de Concepción por las fotografías de microscopio electrónico. Al Proyecto de la Dirección de Investigación de la Universidad de Concepción $\mathrm{N}^{\mathrm{o}}$ 205.113.0681.0, por su apoyo económico en la presente publicación.

\section{RESUMEN}

Se describe el huevo, larva de primer estadio y aparato genital de la hembra de Chabuata castanea con material proveniente de Talcahuano, VIII región, Chile y de huevos obtenidos en laboratorio. Se fotografiaron los huevos con 
microscopia electrónica de barrido para analizar las variaciones entre micropila, celdas primarias y secundarias, concluyéndose que las diferencias permiten una identificación al nivel de especie.

Palabras clave: Chabuata castanea, Hadeninae, estados inmaduros, Talcahuano, Chile.

\section{REFERENCIAS}

Angulo, A.O \& G.Th. Weigert. 1977. Pseudaletia punctulata (Blanchard) y Pseudaletia impuncta (Guenée). Nóctuidos hadeninos similares en Chile (Lepidoptera: Noctuidae). Agro Sur 5: 12-17.

Koehler, P. 1961. Noctuidarum Miscellanea III. Ann. Soc. Cientif. Argentina 172: 69-94.

León, C.A., M.A. Rodríguez \& T.S. Olivares. 2005. Chabuata carneago (Guenée, 1852) y Chabuata castanea (Köhler, 1961) (Lepidoptera: Noctuidae) en Chile. Errores de identificación. Rev. Chilena Ent. 30: 9-16.

Parra L.E, A.O Angulo \& C. Jana-Sáenz. 1986. Lepidópteros de importancia agrícola: clave práctica para su reconocimiento en Chile (Lepidoptera: Noctuidae). Gayana Zool. 50: 81-116.

Pastrana, J.A. 2004. Los lepidópteros argentinos. Sus plantas hospedadoras y otros sustratos alimenticios. South American Biological Control Laboratory USDAARS y Sociedad Entomológica Argentina, Buenos Aires, Argentina. $350 \mathrm{p}$.

Poole, R.W. 1989. Lepidopterum Catalogus (new series). Fascicle 118. Noctuidae Part 2. E.J. Bril/Flora \& Fauna, Nueva York, EEUU. p. 501-1013.

Stehr, F.W. 1987. Order Lepidoptera, p. 288-340. In F.W. Stehr (ed.). Kendall/Hunt, Dubuque, Iowa, EEUU. 